\title{
Design Engineering Research at NIST
}

\section{Kevin W. Lyons}

Peter F. Brown

U.S. DEPARTMENT OF COMMERCE Technology Administration National Institute of Standards

and Technology

Gaithersburg, MD 20899

QC

100

. U56

N0. 5464 



\section{Design Engineering Research at NIST}

\section{Kevin W. Lyons Peter F. Brown}

U.S. DEPARTMENT OF COMMERCE Technology Administration National Institute of Standards and Technology

Gaithersburg, MD 20899

May 1994

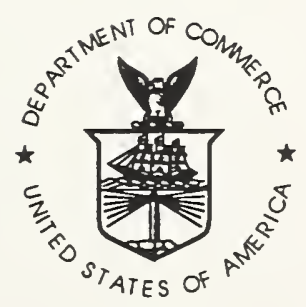

U.S. DEPARTMENT OF COMMERCE Ronald H. Brown, Secretary

TECHNOLOGY ADMINISTRATION Mary L. Good, Under Secretary for Technology

NATIONAL INSTITUTE OF STANDARDS AND TECHNOLOGY

Arati Prabhakar, Director 



\section{Table of contents}

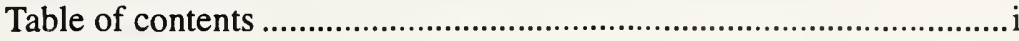

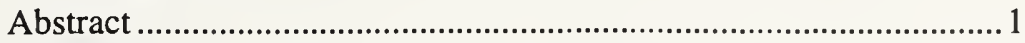

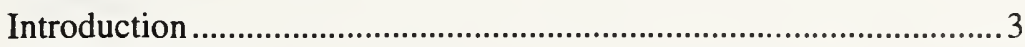

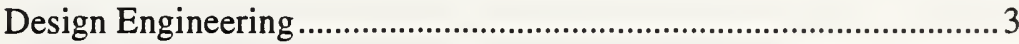

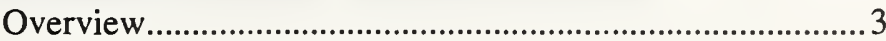

Problem Description/Needs ............................................... 3

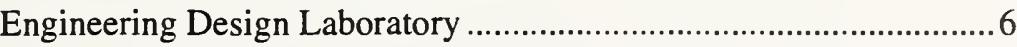

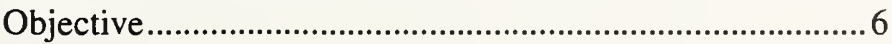

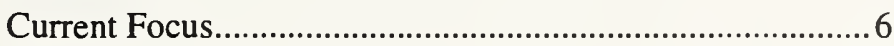

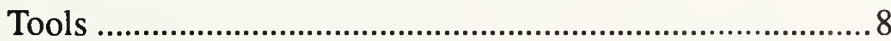

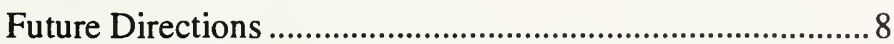

Technology Transfer Program.........................................................

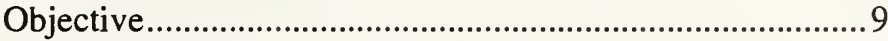

Industry and academia roles ............................................ 10

Collaborative Research Program ........................................ 10

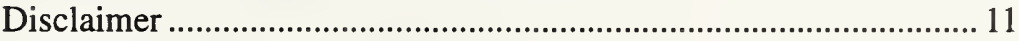

Acknowledgment ....................................................................... 11

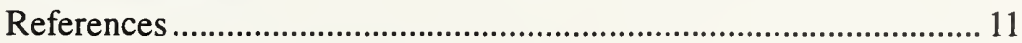

Appendix A - EDL Resources .......................................................... 13

Appendix B - EDL Application Profiles ............................................. 15 


\section{Introduction}

This paper provides an overview of specific design engineering needs and a description of the Engineering Design Lab (EDL) at the National Institute of Standards and Technology. This document is intended to increase the awareness by industrial, academia, and government agencies of the services and facilities offered by the EDL. A primary goal of the EDL is to develop and foster an interest in design engineering and its effects on the product realization cycle. Sufficient interest will ultimately lead to the formation of a design community which will address design engineering concerns and forge a closer working partnership among industry, federal and state agencies, and universities.

\section{Design Engineering}

\section{Overview}

Problem Description/Needs
Companies are now acknowledging that conceptual design not only determines $70 \%^{1}$ of the product costs, but also effects product delivery schedules. In addition, an increased awareness of life-cycle costs and shorter product lives has required companies to better predict and meet product delivery schedules which the marketplace demands. With this increased awareness the design process is coming under increasing scrutiny as product cycle times are being compressed. It is becoming increasingly apparent that a significant portion of a design engineer's time is devoted to performing non-engineering tasks. Designers spend as much of their time being librarians, translators, typists, and programmers as they do being designers. Engineering tools currently available were developed to assist the designer in improving productivity in completing tedious, redundant tasks during the detailed design phase of the product realization process. These tools have evolved to assist in completing design tasks such as drafting, stress analysis, documentation, and specialized simulation tasks. Few tools address methods to assist the designer in the conceptual stage of design or to locate information and knowledge to assist the designer in developing a product.

Taking a successful strategy learned from manufacturing, i.e., just-in-time production, where part and sub-assemblies arrive just as they are needed in the process, we will apply it to the engineering design process. That is, the designer needs to have the right information, at the right time, in a form that can be immediately used. Much of the information that a designer uses is in unstructured non-computer-interpretable representations, such as textbooks, file folders, journal articles, or micro-fiche. This information is, for the most part, stored away and is not easily accessible for timely retrieval and use. When located there are usually other steps needed before the information is in a form that is useful to the designer. All of this "handling" of data and information results in inefficiency and provides a potential source for errors to be introduced in the design process.

To recognize areas within the engineering design process that have a significant impact on the product realization cycle requires that past and current design processes be fully

1 Reference from Improving Engineering Design - Designing for Competitive Advantage study supported by Contract No. DMC-8817926 between the National Science Foundation and the National Academy of Sciences. 
understood and documented. With an understanding of these processes one can then implement changes and track performance by establishing the appropriate metrics based on the process documentation.

Improvements can also be realized by providing the designer with advanced tools to access knowledge and information sources. This information could be related to the current or previous design processes and is in the form of design libraries, technical information (journals, catalogs, books), or manufacturing databases. These tools need to address methods to locate and retrieve, organize, index, and format the knowledge to meet the needs of the designer. A significant portion of information and knowledge available to the designer is unstructured or semi-structured, which complicates its use until methods are developed to transform the information into a computer-interpretable form.

It is important to gain a better understanding of design knowledge and its impact on the product realization process. This will allow the designer to concentrate on product design tasks rather than computer science or database-oriented tasks. Increasing the time that a designer can focus on true design issues is a difficult goal, yet the benefits such as reducing cost and time-to-market are great.

Design knowledge representation: Designers have available to them a wealth of knowledge and information provided by local libraries, personal libraries, and a variety of electronic informational resources such as on-line databases and knowledge based tools. In addition, access to electronic networks such as Internet and EINet ${ }^{1}$ provides vast amounts of knowledge, information, and services to users which quickly can become overwhelming. The ability to provide knowledge and information that is of immediate use (and value) to the designer makes the designer more productive. Productivity is a key performance factor for industry and efforts that result in increasing productivity levels will receive attention. Since a significant portion of the knowledge and information that is available to a designer is not computer-interpretable, further research is needed in developing methods to represent or provide for "transparent" translation of this knowledge and information. It is important that emphasis be placed on the ability of the computer to correctly interpret the knowledge and information within the domain specific context of each informational source.

Design knowledge capture and history: One method to better understand the design process is to evaluate previous designs and processes (history) and document and evaluate the steps and procedures taken for new design projects (capture). To approach this task there are many questions that should be posed. What knowledge and information should be collected during the design? How will this knowledge and information be used and how should it be represented? What methods should be used to collect this knowledge and information, and what format will be used for retrieval and storage? Once collected how should this knowledge and information be organized for presentation to the designer?

In developing a "design history" there will be a record of the designer's intent, alternatives considered and choices made, and the constraints and restraints encountered with methods to attain conformance. Logging design knowledge and information provides a vehicle that will allow new designs to benefit from previous design knowledge and

1 EINet is an information infra-structure project being developed by MCC (Micro-Electronics and Computer Technology Corporation). 
experience. This enables better design decisions and results in better designs in less time. In addition, the design decisions and intent are traceable and thus can assist others in understanding the design so they can be better able to respond to problems encountered in other phases of the product life cycle.

In order to increase the designer's ability to evaluate historical design knowledge and information there needs to be a means to have the knowledge and information in a computer interpretable format. One method to accomplish this involves the use of previous design concepts to assist in approaching new problems and product designs. Case histories, both successes and failures, are retained for review and used at a later time. This allows the designer to retrieve cases that are similar to the problem at hand and identify differences to enhance development of the new design. If a similar case happens to be marked with potential problems or failures it can be used to reconsider the design approach or alert the designer to develop a solution to the problem areas.

Of particular interest is the review of alternate designs against problem constraints and definitions early in the design cycle where maximum benefits can be achieved. Evaluating several choices will expand the designer's "design domain" and often will serve as a catalyst to "induce" innovation and creativity. This is similar to the concepts of "brainstorming" except that the "design domain" may be derived from a library of designs accessible by queries or a knowledge-based application rather than from a true brainstorming session with co-workers. Often when a designer feels comfortable that an acceptable solution has been found, alternate, more innovative designs can be evaluated that may entail more risk associated with successful completion, but have far greater benefits to the product.

There are many potential benefits to having "design capture and history" which are significant in improving the engineering process. Although the realization of these benefits will be varied depending on a number of factors, they all depend on the acceptance of such tools by industry designers and their perception of the "value-added" to the product/process design process.

Design knowledge retrieval: Today designers are faced with an increasing influx of knowledge and information sources at their disposal to assist in design decisions. At first glance one would think that this is beneficial and that the designer needs to make use of this. A closer look reveals that designers have little time to spend sifting through all the available information to find the information they really need. For this maze of information to be of value to the designer, there is a need for tools to assist the designer in finding the needed knowledge and information.

One method to navigate through all of the knowledge and information is to guide the designers search with indexes. Yet the development of an indexing scheme is complicated by the fact that designers need different views of the information depending on what phase of the design cycle they are in. The information required at the conceptual design phase varies significantly in level of detail (abstraction) from that acquired at detail design phase. The amount and detail level also vary depending on the complexity of the problem, the uncertainty of the problem constraints, and the applicability of the design team's skill and experience to the problem at hand. One solution to address abstraction is the creation of multiple databases encompassing the multiple levels of abstraction. Yet this results in redundancy and introduces potential problems related to information consistency. 
As the amount of information increases and access methods are refined, retrieval methods will gain more prominence and the value placed on new advances in this area will be increased. Without such advances, making new information and databases available to the designer will have little impact on improving productivity and design decisions.

Design knowledge transfer: It is the designer's "comfort zone" that often limits creativity and consideration of alternate solutions to the problem at hand. "Comfort Zone" refers to a specific technical area that a designer has technical confidence (or comfort) in his work and proposed solutions. The designers' interests filter their view and perception of the design and the decisions that they will make. Failure to consistently enlarge this zone results in a "comfort zone" that lacks flexibility. If designers are reluctant to broaden their view of alternate designs, their background and experience becomes an important consideration in determining the final product definition. Designers with knowledge and experience in specific areas will most likely prefer that the "quality of the design" be largely evaluated on constraints and definitions relating to their particular areas of strength. The effect on the enterprise, though, can be deleterious unless there is a balanced view of design decisions and their effects on other departments and organizations.

Knowledge is key to providing to the designer the necessary flexibility to respond to today's changing environment. But this broadening of the designer's scope of knowledge comes at a cost. Most designers can not acquire the depth of knowledge in specific areas to respond to the shrinking product cycles in a timely manner. This again focuses the urgency of providing the designer with tools to access knowledge which assist in making design decisions.

Companies' success will be determined by whether their people have mastered the speed and application of the knowledge...... Motorola - Malcolm Baldridge Award recipient

\section{Objective}

Current Focus

\section{Engineering Design Laboratory}

The Engineering Design Laboratory provides an environment to conduct research on design engineering topics. The lab serves as a vehicle to integrate applications, evaluate new approaches, demonstrate and transfer these capabilities to industry and other agencies and institutes, and support the development of standards related to design. In addition the Engineering Design Lab assists NIST in identifying the best practices and methods to design new products and processes. The work conducted in the lab is dynamic, as is the field of research on design engineering. By providing a structure that enhances responsiveness, the lab can better serve all that will make use of its capabilities and services. A major focus of the lab will be on technology transfer and access to the EDL by the design community. This will allow for broader access and distribution of design engineering information, knowledge, and resources available here at NIST and across the nation.

Design knowledge representation: With the ever increasing quantity of information that is available, the question is not whether the information is available but how the designer can locate the information in a timely manner and receive it in a form that can be used immediately. A major focus of the EDL is to develop an understanding of the different design knowledge and information sources and the types of information they 
provide. The first step in the process of developing design knowledge representations will be to develop a taxonomy of design knowledge sources and elements. Development of classifications and categories will provide a better view of what information a designer is confronted with and provide a platform to further refine and define methods to make the right subsets of this knowledge available to the designer.

The proposed informational taxonomy begins with the following three basic classifications[Lee92]:

- Unstructured - such as natural language formats used in books, catalogs, and journals which are not in a format that is computer-interpretable.

- Semi-structured - such as forms, electronic templates, and electronic notebooks in which portions are in a format that is computer-interpretable.

- Structured - such as object-oriented models, knowledge based systems (KBS), and executable documents such as Mathcad ${ }^{1}$.

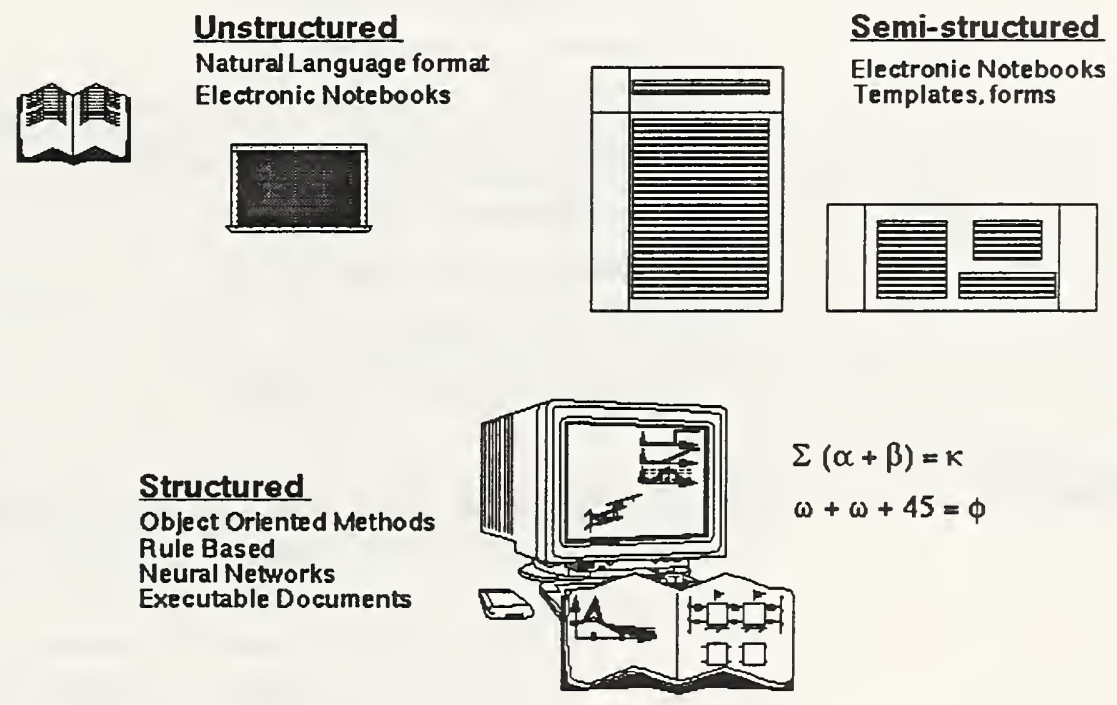

Engineering Tools: The focus of this research is on engineering, fabrication, and assembly problems involving mechanical parts and electro-mechanical assemblies. Research in this area is funded through the ARPA ( $\underline{A}$ dvanced $\underline{R}$ esearch Projects $\underline{A}$ gency) MADE (Manufacturing A utomation and Design Engineering) Project and technical topics include:

- Computational prototyping at various abstraction levels for key engineering analysis

- Intelligent systems engineering approaches to Design for Manufacturing and Assembly (DFMA) through disposal

- Systems engineering frameworks to integrate software tools

- Design libraries and case/model based reasoning approaches

- Manufacturing process modeling and simulation for product/process optimization

- Generative planning of manufacturing and assembly processes

1 An application developed by MathSoft, Inc that provides the capability of combining text and executable math equations within a common page. 
NIST / ARPA role: NIST supports ARPA by assisting in the coordination and execution of the MADE program. The MADE Program is focused on the development of engineering tools and information integration capabilities to support future engineering and manufacturing processes for both defense and commercial products.

NIST's role involves the development of workshops, coordination of meetings, and technical consultation. Technical consultation activities typically involve proposal and project reviews, technical briefings, and evaluation of software. NIST also works with the MADE contractors to develop design ontologies, sharable knowledge bases that support KIF ${ }^{1}$, and in general representations for design knowledge.

The lab currently supports a variety of engineering tools to facilitate research in design engineering. As requirements change the lab will adapt the required applications and methods to provide the needed environment to conduct research. Currently the lab supports tools with the following capabilities (detailed descriptions of specific applications are given in Appendix B):

- Solid Modeling

- Parametric, feature-based

- Finite element analysis (Structural: mechanical components)

- Numerical control (NC) program simulation

- Object-Oriented Modeling

- Translation services (IGES, DXF)

- Design Process Modeling

- Hyper(text/object), multi-media

Future Directions

Design decisions: As designers proceed from the initial product constraints through production of the product there are many decisions that are made. Each of these decisions has an impact on the final product definition. A designer's skill and experience will determine the approach and solutions to problems encountered by the designer. Since most problems faced do not have a clear precise answer, the designer is faced with choosing the "best" solution. This is where experience will often determine whether the optimal or near optimal choice is selected.

One method that will be explored is the use of fuzzy logic in representing imprecise knowledge about a situation. This method allows one to describe an event or fact using statistical methods supporting a certain level or degree of confidence. It is planned to link this research with design knowledge representation. This will provide the designer

$1 \mathrm{KIF}$ (Knowledge Interchange Format) is a formal language for the interchange of knowledge among disparate computer programs. KIF is a programmer-readable language that facilitates the independent development of knowledge manipulating programs. 
an effective decision mechanism to utilize the knowledge that, with proper representation, will be available to the designer.

\section{Design Decisions}

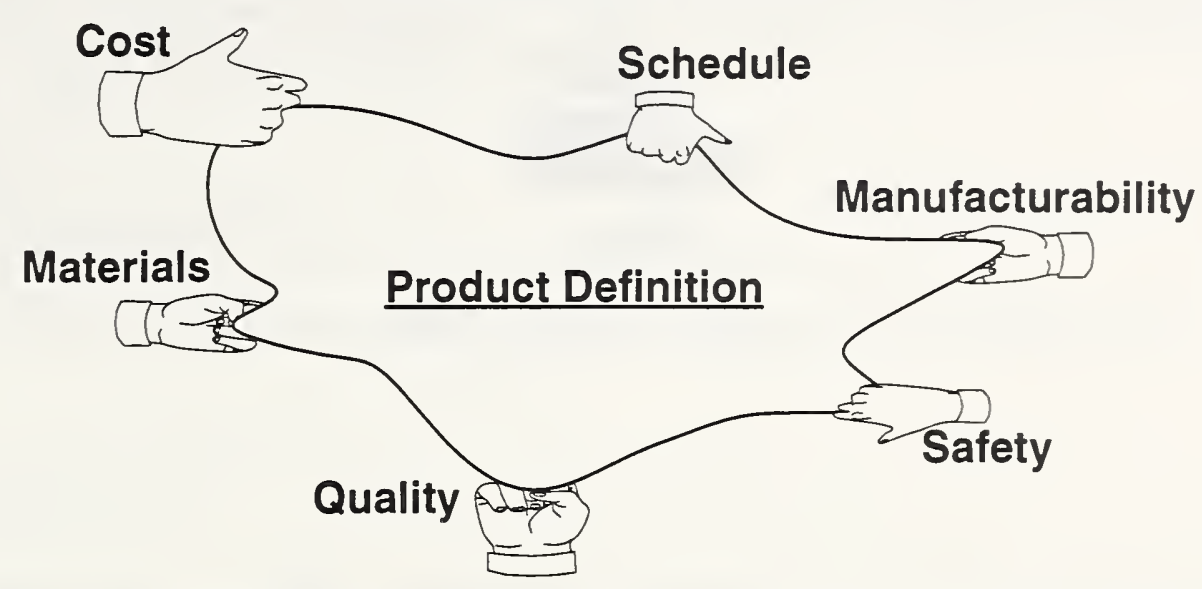

Design for Produceability: The engineering design process culminates in realization of an industrial product. To achieve a more optimal design, which might ultimately determine the product's success or failure, many factors in the whole product life cycle are considered. It is key that these factors be considered at varying abstraction levels throughout the entire product development and manufacturing cycle. Work in this area will focus on "Design for Produceability" (DFP) which encompasses both manufacturing and assembly along with consideration of the manufacturing and assembly technologies and resources available. A "Produceability Repository" is planned that will contain NIST generated knowledge/information and locations of other external sources of knowledge and information related to DFP.

\section{Technology Transfer Program}

NIST has a key role in enabling the transfer of technology to encourage the commercialization of government research efforts. The challenge faced by NIST and other research organizations is to ensure that the research pursued is directed at industrial and commercial current and future needs. With challenge there is always opportunity, and NIST's research programs are structured to address new technologies and the methods to disseminate the knowledge and development work. NIST's strong ties to industry and academic organizations and its leadership capabilities are key factors to transition research to productive commercial use. NIST also recognizes that technology transfer works best when there is a forum that encourages open communications between researchers and the customers that have a need for the technology. NIST's program is designed to support this type of environment and provide assistance on research topics as well as references to other researchers. 
Industry and academia roles

Collaborative Research Program

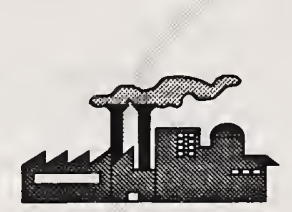

Industrial

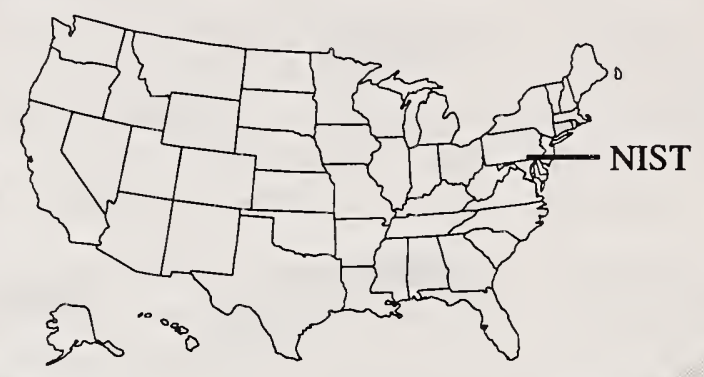

Considerable research is being done related to Design Engineering, yet an often ignored important element of this research is to understand the actual needs of the targeted users. To address this it is key to have industry assistance in developing solutions that are aimed at improving the product realization process. Too often the customer (industry designers) are the forgotten component in establishment of a new tool. This many times results in a technically excellent tool that no one uses. This is not to say that innovation or foresight is to be cast aside, instead what is intended is that the researcher recognize the more immediate and short term needs of industry designers when developing new methods and approaches to improving the engineering design process. This will provide a path to incrementally implement the new tool or method into a production setting. The result is an approach that will provide designers with the tools that they need to accomplish today's problems yet also provide the vision of what can be achieved in the future.

It is crucial that industry and academia work together to accomplish the goal of improving the product realization process. Successful collaboration will achieve significant benefits in improving the productivity of designers in producing products that meet the needs of consumers and in structuring education and design research efforts to allow for continuous process improvement.

In establishing a laboratory for collaboration in design engineering research, attention has been paid to the fact that physical collocation is not a prerequisite for meaningful and productive cooperation. By taking advantage of the current information revolution and its technology, the laboratory will be built as an open, network-accessible facility. Since a major focus of the lab is technology transfer, an effort is under way to provide external access to the EDL by the design community. This will allow for broader access and distribution of design engineering information, knowledge, and resources available here at NIST and at other external locations.

In addition to the research facilitation services described above NIST will have an ongoing research program. The nature of the research will initially address research issues closely related to design engineering knowledge and its representation. 


\section{Disclaimer}

Certain commercial equipment, instruments, or materials are identified in this paper. Such identification does not imply recommendation or endorsement by the National Institute of Standards and Technology, nor does it imply that the products identified are necessarily the best available for the purpose.

\section{Acknowledgment}

The Engineering Design Laboratory is partially funded by the ARPA MADE project.

\section{References}

ARPA 1993 ARPA Manufacturing Automation and Design Engineering (MADE) Program, Broad Agency Announcement BAA \#93-20, Commerce Business Daily, March 15, 1993.

Ray/Feeney 1993 Steven R. Ray, A. Barnard Feeney, “A National Testbed for Process Planning Research,” NIST Interagency Report 5169, 1993.

NAS 1993 National Academy of Sciences, "Toward National Collaboratories: Establishing User-Developer Partnerships," National Academy Press, Washington, D.C., 1993 (in press).

Feeney 1992 A. Barnard Feeney, "User's Guide to the On-Line Bibliographic Reference System for the NIST Process Planning Testbed," NIST Interagency Report 4891, 1992.

Lee 1992 Jintae Lee, "Summary Report of AAAI '92 Workshop on Design Rationale", Center for Coordination Science and AI Laboratory, MIT

Feeney $1991 \quad$ A. Barnard Feeney, "Engineering Design Laboratory Guide," NIST Interagency Report 4519, 1991.

NAP 1991 Committee on Engineering Design Theory and Methodology, Improving Engineering Design - Designing for Competitive Advantage, study supported by Contract No. DMC-8817926 between the National Science Foundation and the National Academy of Sciences.

Gruber/Olsen 1993 Engineering Math Ontologies, Introductory document for Release 0.2 of Stanford's Engineering Math Ontologies. 
References 


\section{Appendix A - EDL Resources}

Below is a listing of the functional capabilities of the Engineering Design Laboratory with a listing of applications currently supported.

\section{Support Tools and Technologies}

\section{Computer systems}

- Sun $^{\dagger}$ Workstations (SPARC 2s, SUN 4 file server)

- Silicon Graphics Workstation (Personal IRIS)

\section{Operating systems}

- $\quad \mathrm{Unix}^{\mathrm{TM}}$ (SunOS 4.1.1, IRIX 4.0.5)

\section{Computer languages}

- $\quad$,, C++, Lucid Common Lisp

\section{Application Systems}

\section{Engineering Design and Solid Modelling Software}

\section{- Pro/ENGINEER ${ }^{\ddagger}$}

\section{Simulation}

- NEWTON $^{* *}$

- $\mathrm{N}-\mathrm{See}^{\dagger \dagger}$

\section{Product and process modelling}

- $\mathrm{CACE} / \mathrm{PM}^{\ddagger \ddagger}$

- OMTool $^{* * *}$

- Mathcad w/Electronic Handbooks ${ }^{\dagger \dagger \dagger}$

* Certain commercial equipment, instruments, or materials are identified in this paper. Such identification does not imply recommendation or endorsement by the National Institute of Standards and Technology, nor does it imply that the products identified are necessarily the best available for the purpose.

$\dagger$ Sun Microsystems, Inc.

$\ddagger$ Pro/ENGINEER is a registered trademark of Parametric Technology Corporation

** Developed by Cornell University

†† Trademark of Microcompatibles, Inc.

扭 Developed by Perceptronics

*** Trademark of General Electric Company

†† Trademark of MathSoft, Inc. 


\section{Translators}

- Pro/ENGINEER to Initial Graphics Exchange Specification (IGES), CATIA*, and DXF (Data Exchange Format)

\section{Electronic Library Services}

- FTP (file transfer protocol) capabilities

- Serial line access for telephone dial-in, to support file downloads via Kermit and $\mathrm{X}$-modem

- Mail service, for automated distribution of files upon request via electronic mail (email)

- MIME protocol, a multi-medial extension to electronic mail

- LISTSERV service, to support automatic maintenance of email mailing lists and discussion groups, or equivalent.

- WAIS (Wide Area Information Service) utilities for indexing and accessing WAIS databases

- Gopher services, providing browsing capabilities for Gopher systems

- WWW (World wide web) services

- Remote interactive services, including:

IRX database of process planning citations

TTY-oriented contributed process planning software (Unix only)

$\mathrm{X}-11$ based remote operation of selected software applications, as determined throughout the year.

* CATIA is a registered trademark of Dassault Systems

$\dagger$ DXF specification was developed by Autodesk 


\section{Appendix B - EDL Application Profiles}

The Engineering Design Laboratory maintains a variety of engineering design tools. The applications are intended to provide a method to evaluate new and innovative research issues.

\section{Supported Applications}

\section{CACE/PM tool}

CACE/PM is a knowledge-based process modeling and simulation application for specifying, visualizing, analyzing, and redesigning product development processes. CACE/ PM allows for representation of pertinent aspects (e.g., what, who, when, and where) and dependencies of an actual or planned product development process.

\section{Mathcad}

Mathcad is a tool for doing numerical calculations, symbolic math, graphing, and documentation. Mathcad offers electronic handbooks that offers the user the means to take standard equations, formulas and diagrams, include them into a document, and interactively use them for calculating results.

\section{NEWTON}

Newton is a dynamic simulation system developed at Cornell University in 1986 and integrated with geometric modeling at Purdue University. Newton supports collision detection and contact analysis of arbitrarily complex polyhedra. In addition to collision detection, control code can be associated with an articulated body to apply forces. For example, a simulation of a two-cycle internal combustion engine can be simulated by specifying the pressure forces occurring inside the piston chamber.

\section{N-See}

$\mathrm{N}-$ See is a solid model numerical control (NC) verification application. Creates a 3D computer model directly from the $\mathrm{CNC}$ code that is sent to the $\mathrm{CNC}$ mill. Provides visualization of material removal to verify NC program accuracy prior to actually cutting material.

\footnotetext{
* Certain commercial equipment, instruments, or materials are identified in this paper. Such identification does not imply recommendation or endorsement by the National Institute of Standards and Technology, nor does it imply that the products identified are necessarily the best available for the purpose.
} 


\section{OMTOOL}

OMTOOL is an application for developing object-oriented analysis and design models using the Object Modeling Technique (OMT) developed by GE scientists. The OMTOOL model consists of two parts: a logical information model and a geometric image or diagram of the logical model. The logical model contains the underlying descriptions shown by the diagram: the classes, their relationships, and other semantics. The logical model can be used for generation of code for either the development of application software or a database. This logical model is independent of any particular representation, such as the geometric diagram, and can be transmitted or expressed textually as well as geometrically. The geometric diagram is OMTOOL's particular representation of the logical information using a specific set of conventional geometric icons and graphical composition rules. Even within a particular representation format there are an infinite number of ways to lay out a diagram representing a particular logical model. A diagram that best captures the semantics of the logical design yet has an layout that permits the information to be easily perceived and understood is desirable.

\section{Pro/ENGINEER}

Pro/ENGINEER is a feature based mechanical design automation tool that supports the design through manufacturing process. Pro/ENGINEER is a feature based system which allows the user to create models which are combinations of design specific features such as ribs, slots, fillets, holes, and shells. Pro/ENGINEER is parametric based which provides for assignment of symbols to the design dimensions allowing for the designer to relate dimensions and features to one another. Additional family products provide for functionality in areas of assembly, manufacturing engineering, and quality control. Pro/ ENGINEER products supported are ASSEMBLY, DESIGN, DETAIL, DRAFT, FEATURE, INTERFACE, MESH, and SURFACE. 
